\title{
Expression and roles of TIPE2 in autoimmune hepatitis
}

\author{
JINHUA QIAN $^{1}$, ZONGDE MENG $^{2}$, JIACHANG GUAN ${ }^{3}$, ZHIWEI ZHANG $^{1}$ and YANGANG WANG ${ }^{1}$ \\ ${ }^{1}$ Department of Spleen and Stomach Diseases, Hebei Province Hospital of Traditional Chinese Medicine; \\ ${ }^{2}$ Department of Traditional Chinese Medicine, Graduate School, Hebei Medical University, Shijiazhuang, Hebei 050011; \\ ${ }^{3}$ Department of Digestive Tract Medicine, Hebei Yiling Hospital, Shijiazhuang, Hebei 050091, P.R. China
}

Received September 11, 2015; Accepted October 21, 2016

DOI: $10.3892 / \mathrm{etm} .2017 .4050$

\begin{abstract}
Tumor necrosis factor, alpha-induced protein 8-like 2 (TIPE2) is associated with the development of hepatic inflammatory diseases. However, to date, the possible role of TIPE2 in autoimmune hepatitis (AIH) has not been reported. The present study aimed to investigate the expression of TIPE2 in peripheral blood mononuclear cells (PBMCs) of mice with AIH. Furthermore, the liver function, pro-inflammatory cytokine production and hepatic histopathology were examined in TIPE2-deficient mice in order to evaluate whether TIPE2 is involved in the pathogenesis of AIH. A murine model of AIH was induced by treatment with concanavalin A (ConA). The expression of TIPE family members in the PBMCs was examined using reverse-transcription quantitative polymerase chain reaction analysis, while the protein expression of TIPE2 was additionally detected by western blot analysis. The activity of alanine amiotransferase (ALT) and aspartate aminotransferase (AST) in the serum was measured on an automated chemical analyzer to assess liver function. The serum levels of tumor necrosis factor- $\alpha$, interleukin (IL)- 6 and IL-12 were measured using commercial ELISA kits. Hematoxylin and eosin staining was performed to assess hepatic histopathology. The results showed that the expression of TIPE2 was significantly decreased in the mice with AIH. Following ConA-induced AIH, TIPE2-deficient mice had significantly increased serum ALT and AST levels, enhanced production of pro-inflammatory cytokines, as well as more severe hepatic inflammation compared with the wild-type mice. In conclusion, the present study demonstrated, for the first time, that TIPE2 is involved in the pathogenesis of AIH. TIPE2 prevents liver dysfunction and inhibits deleterious inflammatory immune responses after $\mathrm{AIH}$ and may therefore serve as a novel agent for the treatment of AIH.
\end{abstract}

Correspondence to: Professor Yangang Wang, Department of Spleen and Stomach Diseases, Hebei Province Hospital of Traditional Chinese Medicine, 389 Zhongshan Road, Shijiazhuang, Hebei 050011, P.R. China

E-mail: yan_gang_hn@163.com

Key words: autoimmune hepatitis, tumor necrosis factor alpha-induced protein 8 -like 2, liver function, proinflammatory cytokines, hepatic histopathology

\section{Introduction}

Autoimmune hepatitis (AIH) is a chronic inflammatory liver disease characterized by interface hepatitis, increased levels of autoantibodies and immunoglobulins (Igs), as well as liver dysfunction (1-4). The disease has a universal distribution affecting all age groups and both genders, irrespective of the ethnicity of the affected individual (5-10). Although much progress has been made in the understanding of the pathogenesis of AIH in the past few years, the immunological mechanism of AIH remain to be elucidated.

The tumor necrosis factor-alpha (TNF- $\alpha$ )-induced protein-8 like-2 (TIPE2) is a newly identified negative regulator of immunity $(11,12)$. It is highly expressed in hematopoietic cells $(13,14)$, and negatively regulates inflammation through T cell receptor (TCR), Toll-like receptor (TLR), nuclear factor-kappa-B, c-Jun N-terminal kinase and p38 signaling pathways $(11,13,15,16-18)$. The association between TIPE2 and liver diseases has been widely studied (19-24). It has been reported that TIPE2 has important roles in suppressing Hepatitis B virus (HBV) -induced hepatic inflammation. Patients with chronic hepatitis B had significantly reduced expression levels of TIPE2 in their peripheral blood mononuclear cells (PBMCs) compared with the healthy controls (22). Kong et al (23) reported that the TIPE2 mRNA expression was significantly downregulated in chronic hepatitis $\mathrm{C}$ patients, and $\mathrm{HCV}$ may promote chronic hepatitis by inhibiting TIPE2 expression. In patients with acute-onchronic hepatitis B liver failure (ACHBLF) (24), the levels of TIPE2 mRNA correlated with serum total bilirubin and model for end-stage liver disease scores. TIPE2 mRNA levels were significantly upregulated in patients with ACHBLF compared with those in the patients with chronic infection or the healthy controls. Cytotoxic T cell-mediated killing of virus-infected hepatocytes is an important pathogenic process of hepatitis B. Recently, Zhang et al (21) reported that TIPE2 protein negatively regulates HBV-specific CD8 (+) T-cell functions in patients with hepatitis B. This suggests that TIPE2 may activate the destruction of hepatocytes by certain types of blood cell. However, to the best of our knowledge, the expression and the role of TIPE2 in AIH has not yet been reported.

In the present study, a murine model of concanavalin A (ConA)-induced AIH was established, and the expression of TIPE family members was assessed in the PBMCs of these mice. Furthermore, liver function, pro-inflammatory cyto- 
kine production and hepatic histopathology were assessed in TIPE2-deficient mice to evaluate whether TIPE2 is involved in the pathogenesis of AIH.

\section{Materials and methods}

Experimental animals. A total of 72 wild-type (WT) C57BL/6 mice were purchased from Shanghai Slac Laboratory Animal, Co., Ltd. (Shanghai, China). The TIPE2-knockout (TIPE2--) mice with C57BL/6J background were generated as described previously (11). All mice used were male, 8-10 weeks old and weighed $22-25 \mathrm{~g}$. The mice were maintained in individual cages under controlled conditions $\left(22 \pm 2^{\circ} \mathrm{C} ; 55 \%\right.$ humidity; $12-\mathrm{h}$ light/dark cycle), with free access to food and fresh water. All animal experiments were approved by the Ethics Committee of Hebei Province Chinese Medicine Hospital (Shijiazhuang, China) following the Guide for the Care and Use of Laboratory Animals published by the National Institutes of Health (NIH Publication no. 86-23, revised 1986). To induce AIH, the mice were injected with $2 \mathrm{mg} / \mathrm{kg}$ ConA (Sigma-Aldrich; Merck Millipore, Darmstadt, Germany) through the tail vein. Mice in the control group were injected with an identical volume of phosphate-buffered saline (PBS). Each group contained 6 mice.

Collection of PBMCs. Blood samples $(\sim 200 \mu \mathrm{l})$ were obtained via the tail vein at 6,12 and $18 \mathrm{~h}$ after ConA-induced AIH. At $24 \mathrm{~h}$, the mice were sacrificed via cervical dislocation and $\sim 1 \mathrm{ml}$ blood samples were harvested. Peripheral blood mononuclear cells (PBMCs) were isolated using Lymphocyte Separation Solution (Sigma-Aldrich; Merck Millipore) and stored at $-80^{\circ} \mathrm{C}$.

Western blot analysis. The PBMCs were lysed with radioimmunoprecipitation assay buffer (Beyotime Institute of Biotechnology, Shanghai, China) and the total protein concentration was determined using a Bradford Protein Concentration Assay kit (Beyotime Institute of Biotechnology) according to the manufacturer's instructions. A total of $30 \mu \mathrm{g}$ protein was separated by $10 \%$ SDS-PAGE and transferred onto a nitrocellulose membrane (Millipore, Billerica, MA, USA). After blocking with 5\% non-fat milk in Tris-buffered saline containing Tween 20 (TBST) at $4^{\circ} \mathrm{C}$ overnight, the membranes were incubated with the following primary antibodies at $4^{\circ} \mathrm{C}$ overnight: Rabbit polyclonal to TIPE2 (dilution, 1:500; cat. no., ab170258; Abcam, Cambridge, MA, USA), rabbit polyclonal to GAPDH (dilution, 1:2,000; cat. no. ab9485; Abcam). GAPDH antibody was used as an internal control. After washing with TBST, the membranes were incubated with horseradish peroxidase-conjugated goat anti-rabbit IgG (dilution, 1:4,000; cat. no. ab6721; Abcam) at room temperature for $2 \mathrm{~h}$. Detection of the signals was performed using an ECL Western Blotting kit (Pierce Biotechnolgy, Inc., Rockford, IL, USA) by exposure to X-ray films (Phenix Research Products, Candler, NC, USA) for 5 min. Protein was quantified using a ScanJet 4C Flatbed Scanner (Hewlett-Packard, Palo Alto, CA, USA) with NIH Image v1.52 software (rsb.info.nih.gov/nih-image/).

Reverse transcription-quantitative polymerase chain reaction $(R T-q P C R)$. Total RNA was extracted from the PBMCs obtained at $12 \mathrm{~h}$ after ConA injection by using TRIzol (Invitrogen, Carlsbad, CA, USA) according to the manufac- turer's instructions. Reverse transcription was performed with a First Strand cDNA Synthesis kit (Fermentas, Vilnius, Lithuania). Real-time PCR was performed in an ABI 7300 Sequence Detection System with a SYBR-Green PCR kit (both Applied Biosystems; Thermo Fisher Scientific, Inc.). All primers used in this study were purchased from Sangon Biotech Co., Ltd., (Shanghai, China), and the sequences were as follows: TIPE, forward 5'-ACATGATGCGGACAGTGG TA-3 and reverse 5'-AGCTCCTGCCTCTAGGTTCC-3'; TIPE1, forward 5'-TGACCCCAAACACCCTATGT-3' and reverse 5'-TCTCCCAATTCTCCACAACC-3'; TIPE2, forward 5'-TGAAACTCAGGTCCGCTTCT-3' and reverse 5'-tCCTAG TGCTGCCTCCAACT-3'; TIPE3, forward 5'-GGCATTCTC TACCGGAACAA-3' and reverse 5'-GTCCTTGCACTCATG CAGAA-3'. For qPCR, $1 \mu \mathrm{l}$ cDNA, $1 \mathrm{nM}$ primers and $12.5 \mu \mathrm{l}$ 2X SYBR Green were mixed to obtain a final volume of $25 \mu \mathrm{l}$. Thermal cycling was performed as follows: 40 cycles at $95^{\circ} \mathrm{C}$ for $30 \mathrm{sec}, 56^{\circ} \mathrm{C}$ for $30 \mathrm{sec}$ and $72^{\circ} \mathrm{C}$ for $30 \mathrm{sec}$. Relative levels of gene expression were determined using the comparative $\mathrm{Cq}$ method (25) with GAPDH as the control.

Measurement of alanine amiotransferase (ALT) and aspartate aminotransferase (AST). The blood samples were centrifuged at $1,000 \mathrm{x} \mathrm{g}$ at $4^{\circ} \mathrm{C}$ for $15 \mathrm{~min}$, and the serum was extracted and stored at $-20^{\circ} \mathrm{C}$. The levels of ALT and AST in the serum were measured using the ALT/AST Assay Reagent kit (Sichuan Maker Science Technology Co., Ltd, Chengdu, China) according to the manufacturer's instructions with colorimetric evaluation on an Automated Chemical Analyzer (7600; Hitachi, Tokyo, Japan).

Cytokine measurement. The levels of TNF- $\alpha$, interleukin(IL)-6 and IL-12 in the serum were measured using commercial kits (R\&D Systems, Inc., Minneapolis, MN, USA) following the manufacturer's instructions.

Hematoxylin and eosin (HE) staining. The mice were sacrificed at $24 \mathrm{~h}$ after ConA-induced AIH using cervical dislocation method. Liver tissues were fixed in $4 \%$ paraformaldehyde (Beijing Dingguo Changsheng Biotechnology Co., Ltd, Beijing, China) for $24 \mathrm{~h}$ and embedded in paraffin. Tissue blocks were sectioned at $4 \mu \mathrm{m}$ thickness and slices were baked at $60^{\circ} \mathrm{C}$ for $4 \mathrm{~h}$. After removal of the paraffin by using xylene and a graded ethanol series, the sections were incubated with hematoxylin (Beyotime Institute of Biotechnology) for $5 \mathrm{~min}$, followed by washing with water and staining with eosin (Beyotime Institute of Biotechnology) for $1 \mathrm{~min}$. The slides were viewed under a microscope (E200; Nikon Corp., Tokyo, Japan).

Statistical analyses. Statistical analyses were performed using SPSS 19.0 software (International Business Machines, Armonk, NY, USA). Values are expressed as the mean \pm standard deviation. Student's $t$-test was used to evaluate the statistical significance of differences between two groups. $\mathrm{P}<0.05$ was considered to indicate a statistically significant difference.

\section{Results}

Expression of TIPE genes in the PBMCs of mice with AIH. To induce AIH, the WT mice were injected with ConA. $12 \mathrm{~h}$ later, the mRNA expression of TIPE genes in the PBMCs 


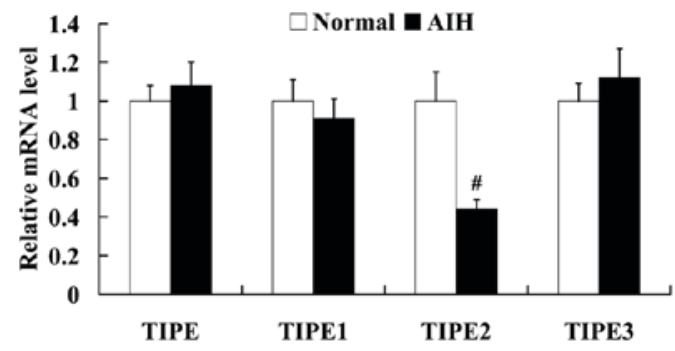

Figure 1. Expression of members of the TIPE family of genes in the peripheral blood mononuclear cells of mice with $\mathrm{AIH}$ at $12 \mathrm{~h}$ after concanavalin A injection. ${ }^{*} \mathrm{P}<0.05$ vs. normal. TIPE, tumor necrosis factor- $\alpha$-induced protein-8; AIH, autoimmune hepatitis.

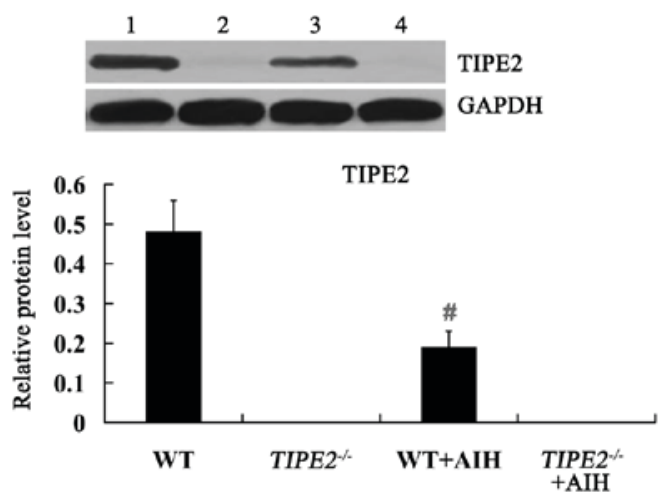

Figure 2. Expression of TIPE2 protein in the PBMCs of TIPE2-deficient mice with AIH at $12 \mathrm{~h}$ after concanavalin A injection. Lanes: 1, WT; 2, TIPE2 $2^{--}$; 3, WT+AIH; 4, TIPE2 ${ }^{--}+\mathrm{AIH} .{ }^{~} \mathrm{P}<0.01$ vs. WT. TIPE2, tumor necrosis factor-alpha-induced protein- 8 like-2; AIH, autoimmune hepatitis; WT, wild-type.

was determined by RT-qPCR analysis. The results revealed that TIPE, TIPE1, TIPE2 and TIPE3 mRNA was detectable in the PBMCs of normal mice. The mRNA expression levels of TIPE2 were significantly decreased in the PBMCs of mice with AIH, while the mRNA expression levels of TIPE, TIPE1 and TIPE3 were not affected (Fig. 1).

Expression of TIPE2 protein in the PBMCs of TIPE2-deficient mice with AIH. The WT and TIPE2 $2^{-1-}$ mice were injected with ConA to induce AIH. $12 \mathrm{~h}$ later, the expression of TIPE2 protein in the PBMCs wsd examined by western blot analysis. As shown in Fig. 2, TIPE2 protein was not detectable in the PBMCs of TIPE2 $2^{-\digamma}$ mice, and that the relative protein levels of TIPE2 were significantly downregulated in the mice with AIH compared with the WT mice.

Expression of ALT and AST in TIPE2-deficient mice with $A I H$. The levels of ALT and AST in the serum were determined in order to evaluate liver function in TIPE2-deficient mice with AIH. As shown in Fig. 3, the levels of ALT and AST were significantly increased from 6 to 24 h post-ConA injection in the WT+AIH group compared with the WT group. Under normal conditions, there was no difference in ALT and AST levels between the WT and the TIPE $2^{-1-}$ mice. However, following ConA-induced AIH, the levels of ALT and AST in TIPE2 $2^{-1-}$ mice were higher compared with those in WT mice.
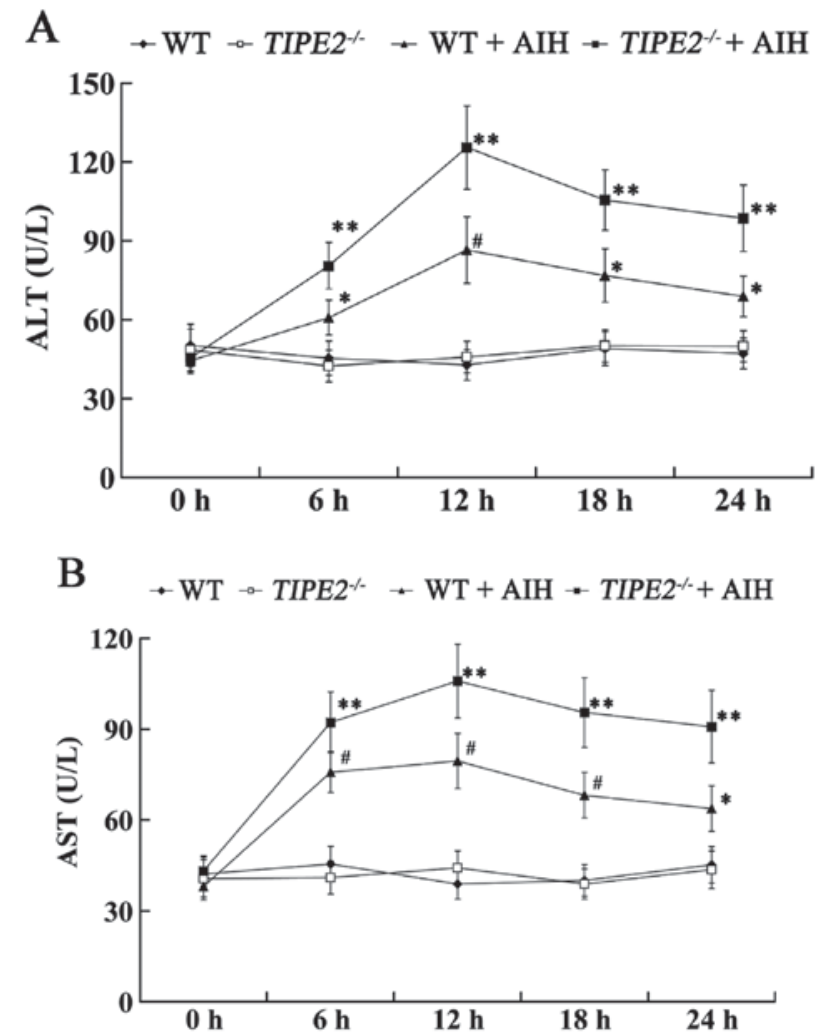

Figure 3. Expression of (A) ALT and (B) AST in the serum of TIPE2-deficient mice with AIH. " $\mathrm{P}<0.05$ and ${ }^{\#} \mathrm{P}<0.01$ vs. WT; ${ }^{* *} \mathrm{P}<0.05$ vs. WT+AIH. TIPE2, tumor necrosis factor-alpha-induced protein-8 like-2; AIH, autoimmune hepatitis; WT, wild-type; ALT, alanine amiotransferase; AST, aspertate aminotransferase.

Expression of pro-inflammatory cytokines in TIPE2-deficient mice with AIH. The expression of pro-inflammatory cytokines, including TNF- $\alpha$, IL- 6 and IL-12 in the serum were measured using ELISA. The expression of these pro-inflammatory cytokines was not significantly different between the WT and the TIPE2 $2^{--}$mice prior to ConA-induced AIH. However, cytokine levels were significantly increased in WT mice following ConA-induced AIH from 6 to $24 \mathrm{~h}$ post-ConA injection. Furthermore, the levels of TNF- $\alpha$, IL- 6 and IL-12 were significantly increased in the TIPE $2^{-1}+\mathrm{AIH}$ group compared with those in the WT+AIH group (Fig. 4).

Hepatic histopathology of TIPE2-deficient mice with AIH. The HE staining of paraffin-embedded liver sections of the experimental mice is shown in Fig. 5. The liver structures of the WT and the TIPE2 $2^{-\digamma}$ mice appeared normal. Following ConA-induced AIH, hepatic injury was shown in the liver tissues. Mononuclear cell infiltration into the parenchyma, portal inflammation and hepatocellular necrosis were identified in the WT and the TIPE $2^{-/}$mice with AIH. The hepatic inflammation was more severe in the TIPE2 $2^{--}+\mathrm{AIH}$ group than that in the WT+AIH group.

\section{Discussion}

The family of TIPE proteins have been recently identified and consists of four members: TIPE, TIPE1, TIPE2 and TIPE3 (26), which have high degrees of sequence homology. 

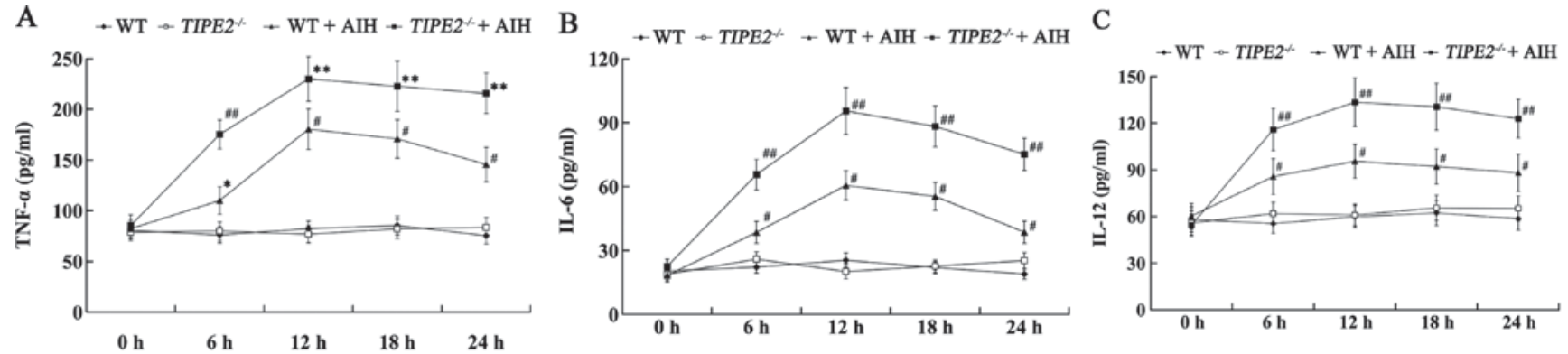

Figure 4. Expression of (A) TNF- $\alpha$, (B) IL-6 and (C) IL-12 in the serum of TIPE2-deficient mice with AIH. ${ }^{*} \mathrm{P}<0.05$ and ${ }^{\#} \mathrm{P}<0.01$ vs. WT; ${ }^{* *} \mathrm{P}<0.05$ and ${ }^{\# \#} \mathrm{P}<0.01$ vs. WT+AIH. TIPE2, TNF- $\alpha$-induced protein-8 like-2; AIH, autoimmune hepatitis; WT, wild-type; TNF- $\alpha$, tumor necrosis factor- $\alpha$; IL, interleukin.

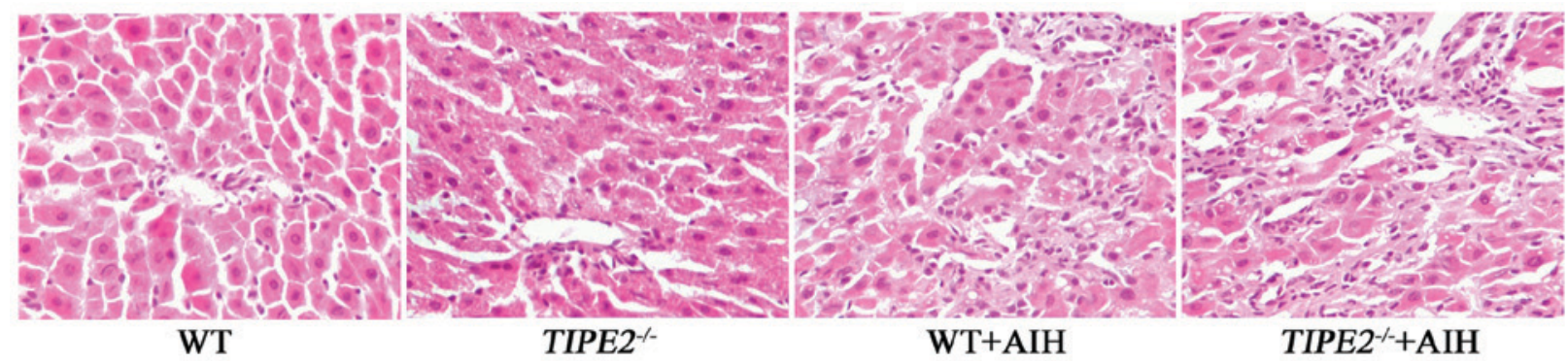

Figure 5. Hepatic histopathology of TIPE2-deficient mice with AIH. The cytoplasm was stained with eosin (red) and the nucleus was stained with hematoxylin (blue). (magnification, x400). TIPE2, tumor necrosis factor-alpha-induced protein-8 like-2; AIH, autoimmune hepatitis; WT, wild-type.

TIPE, the first identified member of this family, acts as a negative mediator of apoptosis and may have a role in tumor progression (12). The biological functions of TIPE1 and TIPE3 in physiological and pathological conditions have remained largely elusive. TIPE1 has been indicated to regulate cell death, and loss of TIPE1 may represent a novel prognostic indicator for hepatocellular carcinoma (27). TIPE3 is regarded as a transfer protein of lipid second messenger that promotes cancer (28). In the present study, the expression of TIPE, TIPE1 and TIPE3 in PMBCs was found to not be affected by ConA-induced AIH in experimental mice. These results indicated that TIPE, TIPE1 and TIPE3 may not be associated with AIH.

TIPE2 is the most widely studied protein of the TIPE family. It has essential roles in maintaining immune homeostasis $(11,15)$. TIPE2 is down-regulated in patients with chronic inflammatory diseases, and TIPE2 deficiency in mice causes fetal inflammatory diseases (11). TIPE2 is also associated with the development of hepatic inflammatory diseases, including as HBV-induced hepatitis, HCV infection and ACHBLF (22-24). However, to the best of our knowledge, the role of TIPE2 in AIH has not been reported to date. In the present study, the expression of TIPE2 was found to be significantly decreased in mice with AIH. These data suggested that TIPE2 may have an important role in the pathogenesis of AIH.

ConA-induced hepatitis is a well-established model for the study of human AIH $(29,30)$, as it mimics numerous aspects of this disease, including liver injury, pro-inflammatory cytokine overproduction (31). In the present study, to further investigate the role of TIPE2 in AIH, TIPE2-deficient mice were treated with ConA. Liver function, pro-inflammatory cytokine production and hepatic histopathology were then examined, revealing that TIPE2-deficient mice had significantly increased serum ALT and AST levels, and enhanced the production of pro-inflammatory cytokines, as well as more severe hepatic inflammation compared with the WT mice, indicating that TIPE2 has a protective effect on liver function after AIH, and that TIPE2 may inhibit pro-inflammatory cytokine overproduction after AIH.

In conclusion, these findings of the present study demonstrated, for the first time, that TIPE2 is involved in the pathogenesis of AIH. The results suggested that TIPE2 is a regulator that prevents liver dysfunction and inhibits a deleterious inflammatory immune response after AIH. The present study suggested that TIPE2 may be a novel target for AIH treatment. Future studies should assess the role of TIPE2 in AIH in clinical studies, comparing the expression of TIPE2 between healthy controls and patients with AIH, and investigating the correlation between TIPE2 expression and clinical indices of patients with AIH.

\section{References}

1. Vergani D and Mieli-Vergani G: Aetiopathogenesis of autoimmune hepatitis. World J Gastroenterol 14: 3306-3312, 2008.

2. Verma S, Maheshwari A and Thuluvath P: Liver failure as initial presentation of autoimmune hepatitis: Clinical characteristics, predictors of response to steroid therapy, and outcomes. Hepatology 49: 1396-1397, 2009.

3. Strassburg CP: Autoimmune hepatitis. Dig Dis 31: 155-163, 2013.

4. Lapierre P, Béland K, Yang R and Alvarez F: Adoptive transfer of ex vivo expanded regulatory $T$ cells in an autoimmune hepatitis murine model restores peripheral tolerance. Hepatology 57: 217-227, 2013.

5. Wong RJ, Gish R, Frederick T, Bzowej N and Frenette C: The impact of race/ethnicity on the clinical epidemiology of autoimmune hepatitis. J Clin Gastroenterol 46: 155-161, 2012. 
6. Schramm C, Kanzler S, zum Büschenfelde KH, Galle PR and Lohse AW: Autoimmune hepatitis in the elderly. Am J Gastroenterol 96: 1587-1591, 2001.

7. Abe M, Mashiba T, Zeniya M, Yamamoto K, Onji M and Tsubouchi H; Autoimmune Hepatitis Study Group-Subgroup of the Intractable Hepato-Biliary Disease Study Group in Japan: Present status of autoimmune hepatitis in Japan: A nationwide survey. J Gastroenterol 46: 1136-1141, 2011.

8. Peng M, Li Y, Zhang M, Jiang Y, Xu Y, Tian Y, Peng F and Gong G: Clinical features in different age groups of patients with autoimmune hepatitis. Exp Ther Med 7: 145-148, 2014

9. Muratori P, Granito A, Quarneti C, Ferri S, Menichella R, Cassani F, Pappas G, Bianchi FB, Lenzi M and Muratori L: Autoimmune hepatitis in Italy: The Bologna experience. J Hepatol 50: 1210-1218, 2009.

10. Chen J, Eslick GD and Weltman M: Systematic review with meta-analysis: Clinical manifestations and management of autoimmune hepatitis in the elderly. Aliment Pharmacol Ther 39: $117-124,2014$

11. Sun H, Gong S, Carmody RJ, Hilliard A, Li L, Sun J, Kong L, $\mathrm{Xu} \mathrm{L}$, Hilliard $\mathrm{B}, \mathrm{Hu} \mathrm{S}$, et al: TIPE2, a negative regulator of innate and adaptive immunity that maintains immune homeostasis. Cell 133: 415-426, 2008.

12. Lou Y and Liu S: The TIPE (TNFAIP8) family in inflammation, immunity, and cancer. Mol Immunol 49: 4-7, 2011.

13. Zhang G, Hao C, Lou Y, Xi W, Wang X, Wang Y, Qu Z, Guo C, Chen Y, Zhang Y and Liu S: Tissue-specific expression of TIPE2 provides insights into its function. Mol Immunol 47: 2435-2442, 2010.

14. Zhang L, Shi Y, Wang Y, Zhu F, Wang Q, Ma C, Chen YH and Zhang L: The unique expression profile of human TIPE2 suggests new functions beyond its role in immune regulation. Mol Immunol 48: 1209-1215, 2011.

15. Zhang X, Wang J, Fan C, Li H, Sun H, Gong S, Chen YH and Shi Y: Crystal structure of TIPE2 provides insights into immune homeostasis. Nat Struct Mol Biol 16: 89-90, 2009.

16. Sun H, Zhuang G, Chai L, Wang Z, Johnson D, Ma Y and Chen YH: TIPE2 controls innate immunity to RNA by targeting the phosphatidylinositol 3-kinase-Rac pathway. J Immunol 189: 2768-2773, 2012

17. Wang Z, Fayngerts S, Wang P, Sun H, Johnson DS, Ruan Q, Guo $\mathrm{W}$ and Chen YH: TIPE2 protein serves as a negative regulator of phagocytosis and oxidative burst during infection. Proc Natl Acad Sci USA 109: 15413-15418, 2012.

18. Lou Y, Liu S, Zhang C, Zhang G, Li J, Ni M, An G, Dong M, Liu X, Zhu F, et al: Enhanced atherosclerosis in TIPE2-deficient mice is associated with increased macrophage responses to oxidized low-density lipoprotein. J Immunol 191: 4849-4857, 2013.
19. Cao X, Zhang L, Shi Y, Sun Y, Dai S, Guo C, Zhu F, Wang Q, Wang J, Wang X, et al: Human tumor necrosis factor (TNF)-alpha-induced protein 8-like 2 suppresses hepatocellular carcinoma metastasis through inhibiting Rac1. Mol Cancer 12: 149, 2013.

20. Zhang YH, Yan HQ, Wang F, Wang YY, Jiang YN, Wang YN and Gao FG: TIPE2 inhibits TNF- $\alpha$-induced hepatocellular carcinoma cell metastasis via Erk1/2 downregulation and NF- $\kappa B$ activation. Int J Oncol 46: 254-264, 2015.

21. Zhang W, Zhang J, Zhao L, Shao J, Cui J, Guo C, Zhu F, Chen YH and Liu S: TIPE2 protein negatively regulates HBV-specific $\mathrm{CD}^{+} \mathrm{T}$ lymphocyte functions in humans. Mol Immunol 64: 204-209, 2015.

22. Xi W, Hu Y, Liu Y, Zhang J, Wang L, Lou Y, Qu Z, Cui J, Zhang G, Liang X, et al: Roles of TIPE2 in hepatitis B virus-induced hepatic inflammation in humans and mice. Mol Immunol 48: 1203-1208, 2011

23. Kong L, Liu K, Zhang YZ, Jin M, Wu BR, Wang WZ, Li W, Nan YM and Chen YH: Downregulation of TIPE2 mRNA expression in peripheral blood mononuclear cells from patients with chronic hepatitis C. Hepatol Int 7: 844-849, 2013.

24. Wang LY, Fan YC, Zhao J, Gao S, Sun FK, Han J, Yang Y and Wang K: Elevated expression of tumour necrosis factor- $\alpha$-induced protein 8 (TNFAIP8)-like $2 \mathrm{mRNA}$ in peripheral blood mononuclear cells is associated with disease progression of acute-on-chronic hepatitis B liver failure. J Viral Hepat 21: 64-73, 2014.

25. Livak KJ and Schmittgen TD: Analysis of relative gene expression data using real-time quantitative PCR and the 2(-Delta Delta C(T)) Method. Methods 25: 402-408, 2001.

26. Gus-Brautbar Y, Johnson D, Zhang L, Sun H, Wang P, Zhang S, Zhang L and Chen YH: The anti-inflammatory TIPE2 is an inhibitor of the oncogenic Ras. Mol Cell 45: 610-618, 2012.

27. Zhang Z, Liang X, Gao L, Ma H, Liu X, Pan Y, Yan W, Shan H, Wang $\mathrm{Z}$, Chen YH and Ma C: TIPE1 induces apoptosis by negatively regulating Rac1 activation in hepatocellular carcinoma cells. Oncogene 34: 2566-2574, 2015.

28. Fayngerts SA, Wu J, Ox ley CL, Liu X, Vourekas A, Cathopoulis T, Wang Z, Cui J, Liu S, Sun H, et al: TIPE3 is the transfer protein of lipid second messengers that promote cancer. Cancer Cell 26: 465-478, 2014

29. Tiegs G, Hentschel J and Wendel A: A T cell-dependent experimental liver injury in mice inducible by concanavalin A. J Clin Invest 90: 196-203, 1992.

30. Shao X, Qian Y, Xu C, Hong B, Xu W, Shen L, Jin C, Wu Z, Tong $\mathrm{X}$ and Yao $\mathrm{H}$ : The protective effect of intrasplenic transplantation of Ad-IL-18BP/IL-4 gene-modified fetal hepatocytes on ConA-induced hepatitis in mice. PLoS One 8: e58836, 2013.

31. Ichiki Y, Aoki CA, Bowlus CL, Shimoda S, Ishibashi H and Gershwin ME: T cell immunity in autoimmune hepatitis. Autoimmun Rev 4: 315-321, 2005. 